PROCEEDINGS OF THE

AMERICAN MATHEMATICAL SOCIETY

Volume 130, Number 2, Pages 413-418

S 0002-9939(01)06104-4

Article electronically published on May 23, 2001

\title{
ON THE STRONG MAXIMUM PRINCIPLE
}

\author{
ARRIGO CELLINA \\ (Communicated by David S. Tartakoff)
}

\begin{abstract}
This paper presents a necessary and sufficient condition on the convex function $f$ in order that continuous solutions to$$
\operatorname{minimize} \int_{\Omega} f(\|\nabla u(x)\|) d x \text { on } u^{0}+W_{0}^{1,1}(\Omega)
$$

satisfy a Strong Maximum Principle on any open connected $\Omega$.
\end{abstract}

\section{INTRODUCTION}

In the present paper we consider properties of solutions to Problem $(\mathrm{P})$ :

$$
\operatorname{minimize} \int_{\Omega} f(\|\nabla u(x)\|) d x \text { on } u^{0}+W_{0}^{1,1}(\Omega) .
$$

Our Theorem 2 below presents a necessary and sufficient condition on the convex function $f$ in order that continuous solutions to (P) satisfy a Strong Maximum Principle on any open connected $\Omega$. Theorem 1 (instrumental to the Proof of the main result) is instead a Comparison Principle, or a weak Maximum Principle, for solutions to $(\mathrm{P})$.

\section{MAIN RESULTS}

In what follows we shall deal with an extended valued, lower semicontinuous, rotationally invariant, convex integrand $F(\xi)=f(\|\xi\|)$ such that $F(0)=0$. By a solution to Problem (P) we shall always mean a function giving a finite value to the integral $\int_{\Omega} f(\|\nabla u(x)\|) d x$. We shall say that the integrand $f$ has the Strong Maximum Principle Property if for any open connected $\Omega$, for every continuous non-negative solution to Problem $(\mathrm{P}), u\left(x^{0}\right)=0$ for some $x^{0} \in \Omega$ implies $u(x) \equiv 0$ on $\Omega$. In this formulation, it is actually a Strong Minimum Principle.

It will simplify our notations to assume that $f$ is extended as 0 for negative values of $t$. In the case $f(t)=0$ for $t=0,=+\infty$ for $t>0$, any function $u$ giving a finite value to the above integral must be such that a.e. $\nabla u(x)=0$. In this case the maximum principles are trivially satisfied, on a connected $\Omega$, and we will not consider this case further. The subdifferential of $f, \partial f$, is a maximal monotone map. Since we have excluded that $\partial f(0)=[0,+\infty), \partial f(t)$ must be defined for $t$ in

Received by the editors March 2, 2000 and, in revised form, June 13, 2000.

1991 Mathematics Subject Classification. Primary 35B50, 49N60.

Key words and phrases. Strong Maximum Principle, Comparison Theorem.

(C)2001 American Mathematical Society 
some interval $[0, \tau)$, otherwise we would contradict maximality. We will call face a convex extremal subset of epi(F). We shall say that an open set $\Omega$ is regular if it has the segment property [2], 4], i.e. it is such that, for every $x^{0} \in \partial \Omega$, there exists a neighborhood $U^{0}$ of $x^{0}$ and a non-zero vector $k$ such that $x+t k \in \Omega$ whenever $x \in \bar{\Omega} \cap U^{0}$ and $t \in(0,1)$. We shall say that $u^{0}(x) \geq w(x)$ (or, equivalently, that $\left.u^{0}(x)-w(x) \geq 0\right)$ for $x \in \partial \Omega$ in the sense of $W^{1,1}(\Omega)$ if $\left(u^{0}(x)-w(x)\right)^{-} \in W_{0}^{1,1}(\Omega)$. The Euclidean norm is $\|$.$\| and the scalar product \langle.,$.$\rangle . We shall denote by B_{1}$ the ball $\{x:\|x\| \leq 1\}$ and by $A_{\alpha, \beta}\left(x^{0}\right)$ the annulus $\alpha<\left\|x-x_{0}\right\|<\beta$ for $0<\alpha<\beta$. Comparison Theorems such as Theorem 1 have been considered by the author in [1], for an integrand of the form $f(p)$, and by Mariconda and Treu, in [3], in the more general setting of an integrand of the form $f(x, u, p)$.

Theorem 1. Let $\Omega$ in $\mathbb{R}^{N}$ be regular; let $\alpha$ and $\beta$ be positive constants such that $\Omega \subset A_{\alpha, \beta}\left(x^{0}\right)$. Let $f: \mathbb{R} \rightarrow \mathbb{R}^{+}$be a (possibly extended valued) lower semicontinuous, convex function, such that $f(0)=0$ and $f(t)>0$ for $t>0$. Let $u^{0}(x)$ be in $W^{1,1}(\Omega)$, and let $w$ be a solution to problem $(\mathrm{P})$ yielding a finite value for $\int_{\Omega} f(\|\nabla w(x)\|) d x$. Let $H$ be a non-negative real and $r \rightarrow R_{H}(r), r$ in $[\alpha, \beta]$, be differentiable and such that $H / r^{N-1} \in \partial f\left(\left|\frac{d}{d r} R_{H}(r)\right|\right)$.

Assume that for $x$ in $\partial \Omega$, we have $u^{0}(x) \geq R_{H}\left(\left\|x-x^{0}\right\|\right)$ in the sense of $W^{1,1}(\Omega)$. Then, a.e. in $\Omega, w(x) \geq R_{H}\left(\left\|x-x^{0}\right\|\right)$.

Notice that when $H=0$ and $\Omega$ is bounded, $\Omega$ is contained in some $A_{\alpha, \beta}\left(x^{0}\right)$ and we obtain the same result as in [1], applied to the case of rotationally invariant integrands $f$. Since the case $H=0$ is considered in [1], we shall assume $H>0$.

Proof. The Theorem is a special case of Theorem 3.14 of 3 . Its proof is also a minor modification of the Proof of Theorem 1 of [1] and it will be only sketched here.

Sketch of the Proof. It is convenient to set $S_{H}(x)$ to be $R_{H}\left(\left\|x-x^{0}\right\|\right)$.

a) Set $E^{-}=\left\{x \in \Omega: w(x)<S_{H}(x)\right\}$. We wish to prove that $E^{-}$has measure zero. Set $\eta^{-}$to be $\min \left\{w-S_{H}, 0\right\}$; then $\eta^{-} \in W_{0}^{1,1}(\Omega)$ and we have

$$
\begin{gathered}
\left(w-\eta^{-}\right)(x)= \begin{cases}w(x) & \text { if } w(x) \geq S_{H}(x), \\
S_{H}(x) & \text { otherwise, }\end{cases} \\
\nabla\left(w-\eta^{-}\right)(x)= \begin{cases}\nabla w(x) & \text { if } w(x) \geq S_{H}(x), \\
\nabla S_{H}(x) & \text { otherwise, }\end{cases}
\end{gathered}
$$

so that

$$
\begin{array}{r}
\int_{\Omega}\left[f(\|\nabla w(x)\|)-f\left(\left\|\nabla\left(w(x)-\eta^{-}(x)\right)\right\|\right)\right] d x \\
=\int_{E^{-}}\left[f(\|\nabla w(x)\|)-f\left(\left\|\nabla S_{H}(x)\right\|\right)\right] d x .
\end{array}
$$

From the convexity of $f$ we have that

$$
f(\|\nabla w(x)\|) \geq f\left(\left\|\nabla S_{H}(x)\right\|\right)+\left\langle p(x), \nabla w(x)-\nabla S_{H}(x)\right\rangle
$$


for any selection $p$ from $x \rightarrow \partial F\left(\nabla S_{H}(x)\right)$, hence in particular for $p_{H}(x)=$ $\frac{H}{\left\|x-x^{0}\right\|^{N-1}} \frac{\left(x-x^{0}\right)}{\left\|x-x^{0}\right\|}$. Therefore

$$
\begin{gathered}
\int_{E^{-}}\left[f(\|\nabla w(x)\|)-f\left(\left\|\nabla\left(w(x)-\eta^{-}(x)\right)\right\|\right)\right] d x \\
\geq \int_{E^{-}}\left\langle p_{H}(x), \nabla w(x)-\nabla S_{H}(x)\right\rangle d x .
\end{gathered}
$$

b) We claim that

$$
\int_{E^{-}}\left\langle p_{H}(x), \nabla w(x)-\nabla S_{H}(x)\right\rangle d x=0 .
$$

Proof of this claim. As in [1], Theorem 1, let $\left(\psi_{n}\right), \psi_{n} \in C^{\infty}(\bar{\Omega})$ and $\psi_{n}(x) \leq 0$ for $x$ in $\partial \Omega$ be a sequence converging to $\left(w-S_{H}\right)$ in $W^{1,1}(\Omega)$ (the assumption of regularity of $\Omega$ is used here). Set $w_{n}=S_{H}+\psi_{n}$ and set $E_{n}$ to be $\{x \in \Omega$ : $\left.w_{n}(x)-S_{H}(x)<0\right\}=\left\{x \in \Omega: \psi_{n}(x)<0\right\}$. To prove the claim it is enough to show that $\int_{E_{n}}\left\langle p_{H}(x), \nabla w_{n}(x)-\nabla S_{H}(x)\right\rangle d x=0$. Passing to polar coordinates $\{\omega, r\}$, the intersection of a half line $L_{c}=\{\omega=c, r \geq 0\}$ with the open set $E_{n}$ can be described as $\left\{\omega=c ; r \in \bigcup_{i}\left(\alpha_{i}(c), \beta_{i}(c)\right)\right\}$ where some or all of the $\left\{\omega=c ; \alpha_{i}(c)\right\}$ and of the $\left\{\omega=c ; \beta_{i}(c)\right\}$ can belong to $\partial \Omega$. We have

$$
\begin{gathered}
\int_{E_{n}}\left\langle p_{H}(x), \nabla\left(w_{n}(x)-S_{H}(x)\right)\right\rangle d x \\
=\int_{\|\omega\|=1}\left(\sum_{i} \int_{\alpha_{i}(c)}^{\beta_{i}(c)} \frac{H}{r^{N-1}}\left(\frac{d}{d r}\left(w_{n}(r, \omega)-v(r)\right)\right) r^{N-1} d r d \omega .\right.
\end{gathered}
$$

For each $i$, when $\left\{\omega=c ; \alpha_{i}(c)\right\}$ is in $\Omega, w_{n}$ and $S_{H}$ coincide and the same is true for $\left\{\omega=c ; \beta_{i}(c)\right\}$. At $\left.\partial \Omega, w_{n}(x) \leq S_{H}(x)\right)$. When $\left\{\omega=c ; \alpha_{i}(c)\right\} \in \partial \Omega,\left\{\omega=c ; \alpha_{i}(c)\right\}$ is the limit of points where $w_{n}(x)>S_{H}(x)$. The same remark applies at the points $\left\{\omega=c ; \beta_{i}(c)\right\}$. Hence we obtain that the last integral is zero.

c) From a) and b) we have

$$
\int_{E^{-}}\left[f(\|\nabla w(x)\|)-f\left(\left\|\nabla S_{H}(x)\right\|\right)\right] d x \geq 0 .
$$

On the other hand, since $w$ is a solution,

$$
\begin{aligned}
\int_{E^{-}}[f(\|\nabla w(x)\|)-f(\| \nabla & \left.\left.S_{H}(x) \|\right)\right] d x \\
& =\int_{\Omega}\left[f(\|\nabla w(x)\|)-f\left(\left\|\nabla\left(w(x)-\eta^{-}(x)\right)\right\|\right)\right] d x \leq 0,
\end{aligned}
$$

so that we infer

$$
\int_{E^{-}}\left[f(\|\nabla w(x)\|)-f\left(\left\|\nabla S_{H}(x)\right\|\right)\right] d x=0 .
$$

d) From the previous results we have

$$
\int_{E^{-}}\left\{f(\|\nabla w(x)\|)-\left[f\left(\left\|\nabla S_{H}(x)\right\|\right)+\left\langle p_{H}(x), \nabla w(x)-\nabla S_{H}(x)\right\rangle\right]\right\} d x=0
$$

so that, being the integrand non-negative, we obtain that a.e. in $E^{-}$,

$$
f(\|\nabla w(x)\|)=f\left(\left\|\nabla S_{H}(x)\right\|\right)+\left\langle p_{H}(x), \nabla w(x)-\nabla S_{H}(x)\right\rangle
$$


and we recall that $p_{H}(x) \in \partial F\left(\nabla S_{H}(x)\right)$. Hence, $\nabla w(x)$ belongs a.e., to the same face of the convex function $\xi \rightarrow f(\|\xi\|)$ that contains $\nabla S_{H}(x)$. These faces are either the extremal points or the one-dimensional faces corresponding to intervals where $f$ is affine, i.e. where $f^{\prime}$ is constant. The function $H / r^{N-1}$, a selection from $\partial f\left(\left|\frac{d}{d r} R_{H}(r)\right|\right)$, is strictly monotonic, so it can coincide at most on a set $Z$ of $\mathbb{R}$ of measure zero with the (at most countable) set of values of the derivative of $f$ corresponding to intervals where $f$ is affine. Hence, $\nabla w(x)$ and $\nabla S_{H}(x)$ can differ only when $x$ belongs to the set of measure zero $\Omega \cap\left\{x:\left\|x-x^{0}\right\| \in Z\right\}$. Hence, $\nabla w(x)$ and $\nabla S_{H}(x)$ for a.e. $x$ are such that $(\nabla w(x), f(\|\nabla w(x)\|))$ and $\left(\nabla S_{H}(x), f\left(\left\|\nabla S_{H}(x)\right\|\right)\right)$ are the same extremal point, i.e. $\nabla w(x)=\nabla S_{H}(x)$ a.e. in $E^{-}$. Hence $\nabla \eta^{-}=0$ a.e. in $\Omega$ and, since $\eta^{-} \in W_{0}^{1,1}(\Omega), E^{-}$has measure zero.

Theorem 2. Let $f: \mathbb{R} \rightarrow \mathbb{R}^{+}$be a (possibly extended valued) lower semicontinuous, convex function such that $f(0)=0$. Then $f$ has the Strong Maximum Principle Property if and only if both conditions i) $\partial f(0)=\{0\}$ and ii) $(\partial f)^{-1}(0)=\{0\}$ hold true.

Proposition. Let $f$ be as in Theorem 2; then conditions i) and ii) are equivalent to:

j) $\lim _{t \rightarrow 0^{+}} f(t) / t=0$,

jj) $f(t)>0$ for $t>0$.

Proof. From the assumptions on $f, \partial f(0) \supset\{0\}$ and $(\partial f)^{-1}(0) \supset\{0\}$.

Assume j) and jj). By jj), $(\partial f)^{-1}(0)$ does not contain $t$ for $t>0$. By j), $\partial f(0)=\{0\}$.

Assume i) and ii). By convexity, $\lim _{t \rightarrow 0^{+}} f(t) / t$ exists. If it is strictly positive, $\partial f(0)$ does not reduce to $\{0\}$. If for some $t>0, f(t)=0$, then $(\partial f)^{-1}(0) \ni t$.

Proof of Theorem 2. Necessity. Assume that the Strong Maximum Principle property holds.

a) From the convexity of $f$ we infer that $\lim _{t \rightarrow 0^{+}} f(t) / t$ exists. We claim that it cannot be that this limit $\ell$ is positive. Assume the contrary. Then, $\partial f(0)=[0, \ell]$ while for $F(\xi)=f(\|\xi\|)$ we have that $\partial F(0)=\ell B_{1}$ and $\partial F(\xi)=\partial f(\|\xi\|) \frac{\xi}{\|\xi\|}$ for $\|\xi\| \neq 0$. Certainly, it cannot be that the function $f$ is $f(t)=\ell t$, for $t \geq 0$. In this case, in fact, we have that the map $v(x)=x_{1} \chi_{\left\{x_{1} \geq 0\right\}}(x)$ is a (non-negative) solution to the problem of minimizing

$$
\int_{C} \ell\|\nabla u(x)\| d x \text { on: } v+W_{0}^{1,1}(C)
$$

where $C$ is the cube $\left\{x:\left|x_{i}\right| \leq 1\right\}$, and the Strong Maximum Principle is violated. To check that $v$ is actually a solution, consider that the vector $p=(\ell, 0, \ldots, 0)$ belongs to the subdifferential of the function $\xi \rightarrow \ell\|\xi\|$ both at $\xi=0$ and at $\xi=(1,0, \ldots, 0)$, i.e. $p$ is a (constant) selection from $x \rightarrow \partial(\ell\|\nabla v(x)\|)$, so that, for any $\eta \in W_{0}^{1,1}(C)$, one has

$$
\int_{C} \ell\|\nabla v(x)+\nabla \eta(x)\| d x \geq \int_{C}(\ell\|\nabla v(x)\|+\langle p, \nabla \eta(x)\rangle) d x=\int_{C} \ell\|\nabla v(x)\| d x .
$$

So the case $f(t)=\ell t(t \geq 0)$ is excluded. This fact implies that the range of the subdifferential of $f$ must contain the interval $[0, \ell+\varepsilon]$ for some positive $\varepsilon$.

Set $\alpha$ such that $1 /(\alpha)^{N-1}=\ell+\varepsilon$; set $r_{1}$ such that $1 / r_{1}^{N-1}=\ell$; set $\beta=2 r_{1}$. We have $\alpha<r_{1}<\beta$. On $[\alpha, \beta]$, the map $1 / r^{N-1}$ takes its values on $(0, \ell+\varepsilon]$ 
and $(\partial f)^{-1}\left(1 / r^{N-1}\right)$ is defined. Set $-d(r)$ to be a (measurable, non-negative) selection from $(\partial f)^{-1}\left(1 / r^{N-1}\right)$, so that $1 / r^{N-1}$ is a selection from $\partial f(-d(r))$. Since $1 / r^{N-1}<\ell$ for $r \in\left[r_{1}, \beta\right]$, we have that $-d(r)=0$ on $\left[r_{1}, \beta\right]$. Since $1 / r^{N-1}>\ell$ on $\left[\alpha, r_{1}\right)$, we have that $-d(r)>0$ there.

Set $v(r)$ to be the non-increasing function

$$
v(r)=0+\int_{\beta}^{r} d(\rho) d \rho .
$$

Then $v(r)=0$ for $r \in\left[r_{1}, \beta\right]$ and $v(\alpha)=\int_{\alpha}^{r_{1}}-d(r) d r>0$. Moreover, $1 / r^{N-1} \in$ $\partial f\left(-v^{\prime}(r)\right)=\partial f\left(\left|v^{\prime}(r)\right|\right)$. Set $A$ to be the annulus $A_{\alpha, \beta}(0)$ and $V(x)=v(\|x\|)$, so that $\nabla V(x)=v^{\prime}(\|x\|) \frac{x}{\|x\|}=\left|v^{\prime}(\|x\|)\right| \frac{-x}{\|x\|}$. Then $\frac{1}{\|x\|^{N-1}} \frac{-x}{\|x\|}$ is a selection from $\partial F(\nabla V(x)), x \in A$.

Consider the minimization problem

$$
\operatorname{minimize} \int_{A} f(\|\nabla u(x)\|) d x \text { on } u^{0}+W_{0}^{1,1}(A)
$$

where $u^{0}(x)=\int_{\alpha}^{r_{1}}-d(r) d r$ for $\|x\|=\alpha$ while $u^{0}(x)=0$ for $\|x\|=\beta$. We claim that $V$ is a solution to this minimum problem. In fact, let $u$ be in $u^{0}+W_{0}^{1,1}(A)$. From the convexity of $f$, for any selection $p$ from $\partial F(\nabla V(x))$ (in particular, for $\left.p(x)=\frac{1}{\|x\|^{N-1}} \frac{-x}{\|x\|}\right)$, we have

$$
\int_{A} f(\|\nabla u(x)\|) d x \geq \int_{A}(f(\|\nabla V(x)\|)+\langle p(x), \nabla(u(x)-V(x))\rangle) d x,
$$

and, passing to polar coordinates,

$$
\begin{aligned}
\int_{A}\langle p(x), \nabla(u(x)-V(x))\rangle & d x \\
= & \int_{\|\omega\|=1} \int_{\alpha}^{\beta} \frac{1}{r^{N-1}}\left(\frac{d}{d r}(v(r)-u(r, \omega))\right) r^{N-1} d r d \omega=0 .
\end{aligned}
$$

This proves that $V$ is a (continuous, non-negative) solution to the minimization problem $\left(P_{A}\right)$. Since $V(x)=0$ for $\|x\| \in\left[r_{1}, \beta\right]$ and $v(\alpha)>0$, we have reached a contradiction to the validity of the strong Maximum Principle on $A$. Hence $\lim _{t \rightarrow 0^{+}} f(t) / t=0$.

b) It cannot be that $f(t)=0$ on some interval $[0, \lambda]$. If such a $\lambda>0$ exists, set $\Omega$ to be an annulus $A_{\alpha, \beta}(0)$, set $v_{\lambda}^{\prime}(r)$ to be $-\lambda \chi_{[\alpha,(\alpha+\beta) / 2]}(r)$ on $A_{\alpha, \beta}(0)$, and $v_{\lambda}(r)$ to be $\lambda(\alpha+\beta) / 2+\int_{\alpha}^{r} v_{\lambda}^{\prime}(s) d s$; consider the Problem $\left(P_{A}\right)$ where $u^{0}(x)=0$ for $\|x\|=\beta$ and $u^{0}(x)=\lambda(\alpha+\beta) / 2$ for $\|x\|=\alpha$. Set $V(x)=v_{\lambda}(\|x\|)$. Then $f(\|\nabla V(x)\|)=0$ a.e.; hence $V$ is a continuous non-negative solution to $\left(P_{A}\right)$, while $V(x)=0$ for $\|x\|=(\alpha+\beta) / 2$ and $V(x)>0$ for $\|x\|=\alpha$.

Sufficiency. Let $\Omega$ be any connected open set. Let $w$ be a continuous solution to $(P)$, non-negative on $\Omega$, and assume that both $E=\{x \in \Omega: w(x)=0\}$ and $\Omega \backslash E$ are non-empty. Then, being $\Omega=E \cup(\Omega \backslash E)$ connected, there exists $x^{*} \in \Omega$ such that $w\left(x^{*}\right)=0$ and $x^{*}$ is the limit of points where $w$ is positive. Let $\rho>0$ be such that $B_{2 \rho}\left(x^{*}\right) \subset \Omega$ and let $x^{0}$ be such that $w\left(x^{0}\right)>0$ and $\left\|x^{0}-x^{*}\right\|<\rho$. From the continuity of $w$, there exists $0<\alpha<\left\|x^{0}-x^{*}\right\|$ and $k^{*}>0$ such that on $\left\{x:\left\|x-x^{0}\right\|=\alpha\right\}$, we have $w(x) \geq k^{*}$. Set $\beta=\rho$, so that $A_{\alpha, \beta}\left(x^{0}\right) \subset \Omega$, and set $\varepsilon$ to be $k^{*} /(\beta-\alpha)$. 
By assumption, $(\partial f)^{-1}(0)=\{0\}$. Since $z \rightarrow(\partial f)^{-1}(z)$ is upper semicontinuous at 0 , there exists $\delta>0$ such that $\left((\partial f)^{-1}(z)\right.$ is defined and $)(\partial f)^{-1}(z) \subset[0, \varepsilon]$ for $z \in[0, \delta]$. Choose $H>0$ sufficiently small so that $0<\frac{H}{r^{N-1}}<\delta$ for $r \in[\alpha, \beta]$. Hence $(\partial f)^{-1}\left(\frac{H}{r^{N-1}}\right) \in[0, \varepsilon]$.

By assumption, $\partial f(0)=\{0\}$. Since $\frac{H}{r^{N-1}}>0$, we have $(\partial f)^{-1}\left(\frac{H}{r^{N-1}}\right)>0$.

Consider the function $r \rightarrow R_{H}(r)=-\int_{\beta}^{r}(\partial f)^{-1}\left(\frac{H}{r^{N-1}}\right) d r$. We have that $R_{H}(\beta)=0$ and $0<R_{H}(\alpha) \leq(\beta-\alpha) \varepsilon=k^{*}$. Hence, for $x$ in $\partial A_{\alpha, \beta}\left(x^{0}\right)$, $w(x) \geq R_{H}\left(\left\|x-x^{0}\right\|\right)$. Moreover, $R_{H}(r)$ is strictly decreasing, so in particular $R_{H}\left(\left\|x^{*}-x^{0}\right\|\right)>R_{H}(\beta)=0$. Apply Theorem 1 with $\Omega=A_{\alpha, \beta}\left(x^{0}\right)$ to infer that, for all $x$ in $A_{\alpha, \beta}\left(x^{0}\right), w(x) \geq R_{H}\left(\left\|x-x^{0}\right\|\right)$. In particular, $0=w\left(x^{*}\right) \geq$ $R_{H}\left(\left\|x^{*}-x^{0}\right\|\right)>0$. Hence the assumption that both $E$ and $\Omega \backslash E$ are non-empty has led to a contradiction.

\section{ACKNOWLEDGEMENT}

The present version of the paper owes much to the precise and kind remarks of an anonymous referee.

\section{REFERENCES}

[1] A. Cellina, On the Bounded Slope Condition and the validity of the Euler Lagrange equation, SIAM J. Control Optim., to appear.

[2] D. Gilbarg, N.S. Trudinger, Elliptic Partial Differential Equations of Second Order, Springer, Berlin, 1983. MR 86c:35035

[3] C. Mariconda and G. Treu, A comparison principle for minimizers, C.R. Acad. Sci. Paris Sér. I Math. 330 (2000), 681-686. CMP 2000:14

[4] G.M. Troianiello, Elliptic Differential Equations and Obstacle Problems, Plenum Press, New York, 1987. MR 92b:35004

Dipartimento di Matematica e Applicazioni, Università di Milano Bicocca, Viale Sarca 202, 20126 Milano, Italy

E-mail address: cellina@matapp.unimib.it 